rmgard Eisenbach-Stangl:

\section{Privatpolizei gegen Drogen - Eine neue soziale Bewegung}

Im Mai 2002 wurde in Graz - einer im Süden Österreichs gelegenen Stadt mit etwa 250.000 Einwohnern - eine neue Organisation gegründet. Sie wurde von den Mitgliedern der »Freiheitlichen Partei Österreichs« (»FPÖ«), die sie ins Leben riefen, »Verein der Bürger für Schutz und Sicherheit« genannt und als »Bürgerwehr « rasch in der Öffentlichkeit bekannt. Die FPÖ hatte sich bei mehreren vorangehenden Wahlen als Partei präsentiert, die für eine drogenfreie Gesellschaft kämpft. Sie ist seit dem Jahr 2000 an der Regierung beteiligt.

Der primäre Auftrag der Bürgerwehr war die »wuchernde Drogenkriminalität zu stoppen«, aber neben dem »Ausspionieren von potenziellen Drogendealern «, hatte sie auch den »Fahrgastschutz in Straßenbahnen", das "Aufspüren von Betrunkenen in Parks « wahrzunehmen und gegen »rücksichtslose Radfahrer« vorzugehen. Der neuen Organisation gehörten 20 Personen an, die in einheitliche T-Shirts gekleidet und mit Mobiltelefonen und Videokameras ausgestattet waren, Pfeffersprays und anderes sollte folgen. Die Ausrüstung wurde von der Freiheitlichen Partei finanziert. Der Obmann der Bürgerwehr war ein Berufssoldat des Österreichischen Bundesheeres im Rang eines Obersten, dem der Ruf anhaftete, in einem »Verein von SS-Veteranen aktiv« zu sein. Das erste Objekt, das die Bürgerwehr überwachte, war die International Bilingual School von Graz (»Der Standard« 10. Mai 2002 und 11./12. Mai 2002).

Die Proteste dagegen waren zahlreich und heftig: Der Innenminister reklamierte die Drogenfahndung als Aufgabe der Exekutive und damit als Aufgabe von »Profis«, der Bürgermeister von Graz warnte vor der »Bespitzelung und Entzweiung der Gesellschaft «, die Direktorin der Internationalen Schule und die Vertreter ihrer Lehrer und Eltern fühlten sich stigmatisiert und diskriminiert, Bürger der Stadt fürchteten die Beschneidung ihrer bürgerlichen Freiheiten und "persönlichen Rechte« (»Die Krone«, 14. Mai 2002, »Profil«, 21, 2002). Trotz Ablehnung und mangelnder Erfolge wurde die Bürgerwehr kurz nach ihrer Gründung um 10 Personen aufgestockt (»Der Standard«, 28. Mai 2002).

Einen Monat später beschäftigten sich die österreichischen Tageszeitungen abermals mit der Bürgerwehr: Ihr Obmann hatte mit seinen Kameraden »noch einige Bier « in der Kaserne getrun- ken. Auf der Heimfahrt mit dem eigenen Auto kam es zu einem Unfall, bei dem ein Ehepaar leicht verletzt wurde. Der Obmann der Bürgerwehr verweigerte den Alkotest, worauf ihm der Führerschein entzogen wurde. Allerdings bekannte er sich bald darauf zu seiner Schuld, legte die Obmannschaft in der Bürgerwehr nieder und erklärte auch seinen Rücktritt als Gemeinderat und Sicherheitssprecher seiner Partei. (»Der Standard«, 8./9. Juni 2002 und »Falter« 24/ 02).

Das Ende der Bürgerwehr schien am Ende des Sommers 2002 nahe: Ihre Mitglieder, die sich in der Zwischenzeit einer »Fortbildung « in Bayern unterzogen hatten, begannen im August an Freitagabenden durch den größten Park von Graz, den "Stadtpark", zu patrouillieren. Aber schon beim zweiten Mal erhielten sie »Begleitschutz«. Ein Student und seine Freunde hatten den »Fanclub der sich geschützt fühlenden Bürger« gegründet, sie folgten der Bürgerwehr laut jubelnd und rezitierend: »Dank unserer guten Bürgerwehr / gibt's keine bösen Bürger mehr «. Passanten applaudierten. Die Bürgerwehr verließ den Stadtpark nach eineinhalb Stunden, statt nach vier, wie den Freitagabend zuvor. Am folgenden Freitag war der Fanclub gewachsen und durch eine weitere Organisation verstärkt: Durch die uniformierte »Bürgerwehr-Kontrollkommission", die mit einer Videokamera ausgestattet war und überprüfen wollte »ob die Bürgerwehr gut arbeitet«. Diesmal verließ die Bürgerwehr den Stadtpark sofort und kehrte auch nicht mehr zurück (»Der Standard «, 16. September 2002). Sie machte allerdings wenige Wochen später wieder auf andere Weise von sich reden: Eine große Flüchtlingsorganisation, wurde vom neuen Leiter der Bürgerwehr beschuldigt, »Anleitungen zum Rechtsbruch « zu geben (»Der Standard«, 26. September 2002).

\section{Ein einzigartiges, aber kein einmaliges Phänomen}

Die Bürgerwehr ist vermutlich ein einzigartiges, aber kein vereinzeltes Phänomen. Verwandte Erscheinungen waren schon seit geraumer Zeit in Österreich zu beobachten gewesen und sie waren auch in anderen Ländern auszumachen. Dazu einige Beispiele: (1) Im Sommer 2001 hatten Bürgermeister zweier österreichischer Gemeinden der Öffentlichkeit eine neue drogenpolitische Maßnahme vorgestellt: Alle jene, die der Polizei Drogendealer zur Kenntnis brachten, sollten eine finanzielle Belohnung erhalten (»Profil « 21 und 47, 2001). (2) Im Herbst 2001 hatten die Bürger einer anderen Gemeinde dem Innenminister mit Straßenblockaden gedroht. In dieser Gemeinde befindet sich das größte Flüchtlingslager Österreichs, in dem sich seit einigen Jahren auch asylsuchende Schwarzafrikaner aufhalten. Schwarzafrikaner stehen seit längerem unter dem generellen Verdacht, Drogendealer zu sein. Die Bürger hatten folgerichtig das Gefühl entwickelt, der
Drogenhandel nehme in ihrer Gemeinde überhand, sie würden durch die Maßnahmen des Innenministeriums nicht ausreichend geschützt und sie waren in ihren Ängsten von der FPÖ bestärkt worden (»Die Krone«, 7. November 2002). (3) Ein drittes Beispiel aus Österreich: Als die Grazer Bürgerwehr in Aktion trat, forderten Angehörige der Führung der FPÖ, ähnliche Organisationen auch in anderen Städten und Bundesländern zu etablieren (»Der Standard«, 10. Mai 2002).

In den hier zitierten österreichischen Beispielen ist der Kampf gegen den Drogenhandel eng mit mehr oder minder offen geäußerter Fremdenfeindlichkeit verbunden. Zumindest auf den ersten Blick ist dies nicht der Fall im folgenden Beispiel, das aus Frankreich stammt: Als ein Vater von acht Kindern im Sommer 2001 aus den Ferien heimkehrt, entdeckt er, dass sich in dem Bezirk von Paris, in dem er lebt, in der Zwischenzeit Drogendealer - "Fachos" wie er sie nennt niedergelassen haben. Der mehrfache Vater ist ein gebildeter Mann, aber er vertraut dem Staat nicht, er geht nie zu Wahlen und er geht nun auch nicht zur Polizei. Er überzeugt statt dessen seine Nachbarn, mit ihm ein »collectif anticrack « zu gründen, das jeden Mittwochabend durch den Bezirk partroulliert, und so demonstrieren will, dass dieser noch immer seinen alteingesessenen Bürgern gehört. Eine weitere Maßnahme, die das »collectif « erfand, war ein regelmäßiges Treffen unter den Fenstern eines Gebäudes, in dem ihrer Meinung nach, Drogen verkauft wurden (»Le Monde «, 8. Juni 2002).

Haben die hier berichteten Ereignisse einen gemeinsamen gesellschaftlichen Hintergrund? Wenn ja, wodurch zeichnet er sich aus? Und wie lässt sich die Gründung privater Vereine, die gegen den Drogenhandel vorgehen wollen, vor diesem Hintergrund verstehen? Ich möchte im folgenden einige Überlegungen vorstellen, die ebenso wenig systematisch sind, wie die Beispiele, die ich angeführt habe.

\section{Der sozio-politische Kontext}

Eine Erklärung, die geradezu ins Auge springt, lautet in etwa: der Drogenhandel ist dichter und organisierter geworden, er hat nun Regionen erreicht, die zuvor von ihm verschont gewesen waren, die Aufdringlichkeit und Aggressivität der Drogenhändler hat zugenommen und der Drogenkonsum ist risikoreicher geworden, nicht zuletzt weil er mit AIDS und anderen ansteckenden Krankheiten einhergeht. Gegen diese Erklärung lassen sich viele Argumente ins Feld führen - so zum Beispiel die plausible Beobachtung, dass der Organisationsgrad des Drogenhandels aufgrund der intensivierten Verfolgung abgenommen hat -, doch möchte ich sie hier nicht grundsätzlich in Frage stellen, sondern nur gesellschaftliche Bedingungen diskutieren, die zumindest zusätzlich in Betracht zu ziehen sind. 
Eine Gemeinsamkeit von so verschiedenen Organisationen, wie der Bürgerwehr von Graz und dem collectif anticrack des XIX. Bezirks von Paris, ist der schwindende Glaube an den Staat und das nachlassende Vertrauen in seine Schutzmacht. Das ist natürlich eine sensible Wahrnehmung, obwohl sie bei den hier zitierten sozialen Bewegungen auf ein sehr begrenztes Gebiet beschränkt bleibt: Die Instrumente des Nationalstaates waren nie dazu gedacht gewesen, globale Probleme zu bewältigen. Der Nationalstaat ist zunehmend hilflos, wenn die Regulierung internationaler Märkte - seien sie nun legal oder illegal - ansteht.

Wenn angesichts »der Globalisierung « die Ordnungsmacht des Nationalstaates abnimmt und er »schwach « wird, entsteht das Bedürfnis, die Verteidigung auf anderen Ebenen auszubauen und andere Ordnungs- und Schutzmächte zu entwickeln. Gut bekannt sind Organisationen, die auf der Ähnlichkeit der Interessen, Problemlagen und Lebensstile ihrer Mitglieder aufbauen, wie zum Beispiel Vereine von Eltern drogenabhängiger Kinder oder in manchen Ländern auch Vereine von Drogenabhängigen selbst. Im Gegensatz zu ihnen sind die oben beschriebenen Organisationen auf Gemeinsamkeiten aufgebaut, die fast vergessen schienen: Nachbarschaft, räumliche Nähe und teils auch Sprache und Ethnizität. Aber in einer Welt, die unübersichtlich geworden ist, erscheint das eigene Lebensumfeld wohl einerseits als gefährdeter, aber andererseits auch als wertvoller und der Verteidigung würdig.

Die Schutzmächte, die unterhalb der staatlichen Ebene aufgebaut werden, gehen ganz offensichtlich mit einem geringeren Grad an Neutralität gegenüber dem »zu Kontrollierenden « einher und sind von offen feindseligen Haltungen gegen »den Feind « getragen. Staatliche Maßnahmen sind üblicherweise streng geregelt, sie werden von Experten angeleitet und überwacht - bei den zivilen Schutzmächten ist dies in geringerem Maße bis gar nicht der Fall. Der Aufbau und die Ausübung ziviler Verteidigung erlaubt es daher emotionaler zu sein, und dies dürfte wiederum viele anziehen. In Abhängigkeit davon, welche politische Farbe die zivile Schutzmacht und ihre Mitglieder tragen, und auch in Abhängigkeit von den politischen Traditionen in die sie eingebettet sind, ist der Feind einmal ein "facho" und einmal ein schwarzafrikanischer Flüchtling. Aber in jedem Fall scheint der Feind aus sozialen Lagen zu stammen, die jenen, die ihr Lebensumfeld verteidigen, sehr unvertraut sind. Er oder sie ist »das Andere«, das von der Gemeinschaft auszuschließen ist.

Der Aufbau ziviler Schutzorganisationen gegen den Drogenhandel ist auch im Kontext der Risikoprävention zu betrachten, wie sie in industrialisierten Ländern zur Zeit vorwiegend eingesetzt wird. In dieser Form zielt sie auf die Differenzierung von Wahrnehmung, Empfindung und Erleben, wie auf die Steigerung von Aktivität, sie zielt auf die "Ermächtigung « von Einzelnen und sozialen Aggregaten, wie unter anderem von Gemeinden und Organisationen - oft auf Kosten des Verstehens von umfassenderen sozialen Prozessen und politischen Zusammenhängen. Güter, die bisher einen öffentlichen Charakter hatten, wie Gesundheit und Sicherheit, werden in diesem »Verfahren « zu Gütern, die (individuell) hergestellt und gekauft werden können - von Einzelnen, oder doch zumindest von der Gemeinde und den Organisationen, denen man angehört. Dies entspricht teilweise der Realität, bildet sie aber keineswegs zur Gänze ab: die komplexen "globalen « Interdependenzen werden in diesem Verfahren vernachlässigt, wenn sie nicht sogar ausgeschlossen werden müssen.

Die zivile Verteidigung gegen die Drogen kann schließlich auch im Kontext der schwierigen Bildung von Identität betrachtet werden. In einer Konsumgesellschaft muss Identität notgedrungen ein »Konsumentenprofil « umfassen, wenn es nicht sogar eines solchen als Basis bedarf. Ein Werbespruch bringt es auf den Punkt. »Ich konsumiere, also bin ich «. Ein anderer leitet folgendermaßen zum Fremd- und Selbstverständnis an: »Sage mir was Du isst und trinkst, und ich sage Dir, wer Du bist «. Auch die Ablehnung von Konsumgütern informiert darüber, wer man ist. Vermutlich eignen sich die Haltungen gegenüber psychoaktiven Substanzen besonders für die Bildung und Darstellung von Identität. Ein Weintrinker ist anders als ein Biertrinker, ein Marihuanagebraucher anders als einer der Heroin injiziert und einer, der seinen Bezirk gegen Dealer verteidigt, unterscheidet sich von allen anderen. Haltungen zu psychoaktiven Substanzen erlauben es zunehmend auch den politischen Parteien, sich zu differenzieren und ein mehr oder minder scharfes, unterschiedliches Profil zu entwickeln: wie zum Beispiel der FPÖ, die, wie bereits angemerkt, auf eine drogenfreie Gesellschaft setzt.

Aber vielleicht ist auch alles viel einfacher, vielleicht geht es nur darum einen Fuß auf den Boden dieser chaotischen und zunehmend unübersichtlichen Welt zu bekommen, wenn man gegen etwas kämpft, das man als »unserer Lebensweise « nicht zugehörig erlebt. »Our way of life - OWL « hat es Patricia Highsmith in ihrem berühmten Roman «Das Zittern des Fälschers« genannt. Wenn diese Annahme zutrifft, haben wir in der nahem Zukunft mehr und mehr der Erscheinungen $\mathrm{zu}$ erwarten, die oben beschrieben wurden.

Irmgard Eisenbach-Stangl forscht am Ludwig-Boltzmann-Institut für Suchtforschung in Wien ettina Paul:

\section{»Do not disturb!« \\ Der erfolgreiche Transfer privaten Protestes in politische Programme}

Die Entwicklung privater Initiativen, wie sie Irmgard Eisenbach-Stangl für Österreich beschreibt, sind auch in Deutschland ein bekanntes, sogar alltägliches Phänomen. Doch gibt es wesentliche Unterschiede.

Der Zusammenschluss von Bürger/innen zur Erhöhung eines »Sicherheitsgefühls« im eigenen Wohnumfeld wird mit einer diffusen Kriminalitätsbedrohung und dem Wunsch nach Schutz für ihr Eigentum und die körperliche Unversehrtheit ihrer Familien begründet. Diese so genannte »Kriminalitätsfurcht « vereint diverse Ängste, Befürchtungen, Aversionen u.Ä. zu einem Bedrohungsmoment, egal, ob sich die Abneigung dabei gegen den Zustand der Dunkelheit, gegen Personen, unbekannte Lebensstile oder medial-vermittelte Szenarien richtet. Die Tätigkeiten privater Initiativen zeugen von dieser Bedrohungskonstruktion. Im Jahre 2000 wurde in Rahlstedt, einem weitläufigen Stadtteil am Rande Hamburgs, eine Initiative von 40 Bewohner/innen ins Leben gerufen, die sich »Mehr Sicherheit für Rahlstedt e.V.« nennt. Ihre Aktivitäten umfassen abendliche Patrouillen in ihrer (gutbürgerlichen) Nachbarschaft. Ausgerüstet mit Taschenlampen und Handys suchen sie nach verdächtigen Personen oder Begebenheiten. Jede Person, die der Nachbarschaft nicht bekannt ist, steht erst einmal unter Verdacht, vor allem aber solche, bei denen die äußerliche Erscheinung als Gefahrenindiz gewertet wird. Diese Erscheinungen beinhalten für die Gegend unübliche Anzeichen von Armut, Jugendkultur oder kultureller Herkunft. Der Fremdheitsaspekt wirkt bedrohlich, wird mit einem Angriff auf die Gemeinschaft gleichgesetzt. Die Initiative versteht sich dabei als Ergänzung zur Polizei, nicht als deren Ersatz. Als Grund für die Aufnahme ihrer Aktivitäten führt sie an, dass die staatlichen Einsatzkräfte nicht in der Lage seien, sie und ihre Familien ausreichend zu beschützen.

Phänomene, wie die der Rahlstedter Initiative, sind in Deutschland nicht selten, trotzdem hat sich parallel dazu eine weitreichende kommerzielle Sicherheitsindustrie entwickelt, die die meisten privaten Aktivitäten auf diesem Gebiet übernommen hat. Dies ist ebenfalls in diesem Teil Hamburgs zu beobachten. Eine private Sicherheitsfirma fährt dort ebenfalls ihre Patrouillen. Während die verbliebenen privaten Initiativen hauptsächlich am Rande der großen Städte aktiv sind, in 